\title{
The comparison of three media on the in vitro maturation rate of pig oocytes
}

\author{
G.M. Pyoos ${ }^{2,3 \#}$, A.M. Maqhashu' ${ }^{2}$, M.M. Scholtz ${ }^{2,3}$ \& T.L. Nedambale ${ }^{1,2,3,4}$ \\ ${ }^{1}$ Tshwane University of Technology, Department of Animal Sciences, Faculty of Science, Tshwane University of \\ Technology, Private Bag X680, Pretoria, 0001, South Africa \\ ${ }^{2}$ Agricultural Research Council, Animal Production Institute, Private Bag X 02, Irene, 0062, South Africa \\ ${ }^{3}$ University of the Free state, Department of Animal, Wildlife and Grassland Sciences, PO Box 339, Bloemfontein, \\ South Africa \\ ${ }^{4}$ University of Venda, Department of Animal Science, School of Agriculture, Private Bag X5050, Thohoyandou, \\ 0950, South Africa
}

(Received 3 October 2017; Accepted 8 November 2018; First published online 5 December 2018)

\begin{abstract}
In vitro embryo production in pigs remains a challenging obstacle, given the high rate of polyspermy following in vitro fertilization. Improving the efficiency of successful in vitro maturation, which leads to sequential in vitro fertilization and culture, augments the need for modern biotechnologies in pig farming systems, and subsequently conserves germplasm, that is, embryos to be used in future breeding programs. Thus, in this study the effects were compared of North Carolina State University-23 (NCSU-23), North Carolina State University-37 (NCSU-37), and epidermal growth factor (EGF/TCM-199) media on pig oocyte maturation rate and subsequent polar body formations in vitro. Ovaries were collected from a local abattoir in a controlled temperature of $39{ }^{\circ} \mathrm{C}$. The oocytes were aspirated with an 18-gauge needle. Oocytes surrounded by a uniform complex-cumulus mass were selected. A total of 284 oocytes were allocated to the study, and were incubated with $5 \% \mathrm{CO}_{2}$ at $39{ }^{\circ} \mathrm{C}$ for 48 hours. In vitro maturation rate was observed by checking the presence of expanded cumulus-oocyte complex (COC) and polar body formations under a light microscope. The maturation rate and polar body formations were significantly higher in NCSU-37 with $85.9 \%$ and $81.9 \%$, respectively. However, there were no significant differences in EGF and NCSU-23 media as 77.1\%; $72.5 \%$ and $77.1 \% ; 72.7 \%$ were obtained for maturation rate and polar body formations, respectively. In conclusion, EGF and NCSU-23 had lower rates of maturation and meiotic development owing to their dissimilarities in composition. However, NSCU-37 rendered the highest results because the composition of the media contained beneficial constituents, that is, gonadotropins. New assisted reproductive programs may be important for commercial pig farming systems, as this may facilitate innovative ways of production.
\end{abstract}

Keywords: Embryo production, genetic resources, pig production, reproductive biotechnology

\# Corresponding author: pyoosg@arc.agric.za

\section{Introduction}

To date, producing in vitro pig embryos in South Africa has been challenging. Moreover, studies pertaining to in vitro produced embryos have endeavoured to advance the quality of in vitro matured oocytes by improving the maturation media with growth factors (Grupen, 2014). Brüssow et al. (2000) reported that pig ovaries contain an accumulation of primordial follicles (approximately 420,000 ) and this compromises the use of these pools of oocytes for embryo production. Oocytes need to possess meiotic competency as a prerequisite for in vitro fertilization and further embryo development. Furthermore, in vitro conditions during maturation need to be optimal. That is, during incubation, temperature should be between $38{ }^{\circ} \mathrm{C}$ and $39{ }^{\circ} \mathrm{C}$, $\mathrm{CO}_{2}$ gases at a level of $5 \%$, and the time should not exceed 48 hours, as this may denature oocytes or hinder maturation, as described by Petters \& Wells (1993). Therefore, in vitro maturation is important for the development of the first polar body, leading to successive cleavage and embryonic development.

Numerous factors influence nuclear or cytoplasmic maturation of porcine oocytes, as evaluated by maturation to metaphase II, fertilization, male pronuclear formation, and cleavage or blastocyst development. 
These factors include follicular fluid, oviductal fluid, steroids, other types of serum from other animal sources, and gonadotropins (Bolling, 2001).

The maturational (cytoplasmic and nuclear) and developmental (fertilization, pronuclear formation and cleavage) competencies of oocytes are influenced by the presence of follicular fluid and the size of the follicle from which the COC is harvested (Vatzias \& Hagen, 1999). Although Mattioli et al. (1988) were successful in producing piglets from porcine in vitro Matured - in vitro Fertilization (IVM-IFV) procedures, two major concerns prevail in porcine embryo production, such as the high occurrence of polyspermy during in vitro fertilization and the low quality of embryos at the blastocyst stage (Nagai et al., 2006).

South Africa has limited information on the use of IVM-IVF technologies in the commercial pig industry. The potential of implementing the production of embryos in assisted reproductive technologies in the pig industries has not been fully investigated. Currently, the production of viable embryos confronts major concerns, which means that the use of embryo production in the commercial sector faces endless obstacles. It is also important to implement innovative assisted reproductive programs that can provide the foundations of genetic diversity for commercial farming systems, which may facilitate increasing production (Nedambale et al., 2010). In addition, it is important for the conservation of indigenous genetic resources.

Improving the efficiency of successful IVM-IVF procedures aids in implementing modern biotechnologies in farming systems and conserving good-quality embryos for future assisted reproductive programs, such as embryo transfer. To augment cytoplasmic maturation of porcine oocytes, which may lead to higher rates of fertility and increased embryo development and quality in IVM-IVF procedures, this study compared the effects of three standard maturation media and the subsequent extrusion of the polar body as an indicator of maturation in pig oocytes.

\section{Materials and Methods}

Pig ovaries were collected three times a week from an abattoir in Springs, Johannesburg, during the winter. Each pair of ovaries provided an average of 25 oocytes from mixed breeds of unknown origin. Ovaries were collected in a thermos flask, preserved in $0.9 \% \mathrm{NaCl}$ saline solution, and maintained at a temperature of $39{ }^{\circ} \mathrm{C}$ to prevent further contamination and denaturing of oocytes from cold shock. Ovaries were transported to the laboratory within one hour of slaughter. Oocytes were aspirated from medium-size follicles ( $3 \mathrm{~mm}$ to $6 \mathrm{~mm}$ in diameter) on the surface of the ovaries with an 18-gauge needle fixed to a $10 \mathrm{~mL}$ disposable syringe. Oocytes surrounded by a uniform complex-cumulus mass and with a consistently granulated cytoplasm were selected and washed three times in standard washing media-199 (M199), supplemented with $10 \%$ foetal bovine serum.

Groups of 80 to 100 oocytes were randomly allocated to each well of a four-well multi-dish (Thermo Fisher Scientific ${ }^{\mathrm{TM}}$ ), holding $500 \mu \mathrm{L}$ maturation media, covered with $250 \mu \mathrm{L}$ pre-warmed mineral oil (Sigma, Germany) to prevent the maturation media from evaporating, and equilibrated in an incubation system supplemented with $5 \% \mathrm{CO}_{2}$ gas at approximately $39{ }^{\circ} \mathrm{C}$ for 48 hours. Oocytes were matured in standard EGF media for 48 hours, and in NCSU-23 and NCSU-37 media for 24 hours in the first solution, and another 24 hours in a second solution for NCSU-23, following the protocol of Petters \& Wells (1993) and NCSU-37 as described by Funahashi \& Day (1997). The compositions of standard EGF, NCSU-23, and NCSU-37 maturation media are given in Tables 1, 2 and 3, respectively.

Table 1 Composition of standard epidermal growth factor/Tissue Culture Medium-199 maturation media (20 $\mathrm{mL})$

\begin{tabular}{lc}
\hline Constituent & Concentration \\
\hline Media $199+10 \%$ BSA & $20 \mathrm{~mL}$ \\
EGF & $40 \mu \mathrm{L}$ \\
Na Pyruvate & $20 \mu \mathrm{L}$ \\
PFF (porcine follicle fluid) & $2 \mathrm{~mL}$ \\
\hline
\end{tabular}

BSA: bovine serum albumin, EGF: epidermal growth factor 
Table 2 Composition of North Carolina State University-23 maturation media (50 mL)

\begin{tabular}{lc}
\hline Constituent & Concentration \\
\hline Stock solution A & $36 \mathrm{~mL}$ \\
Stock solution B & $9 \mathrm{~mL}$ \\
PFF & $5 \mathrm{~mL}$ \\
Glucose & $0.0045 \mathrm{~g}$ \\
Sorbitol & $0.0984 \mathrm{~g}$ \\
B-ME stock solution & $50 \mu \mathrm{L}$ \\
L-Cysteine stock solution & $500 \mu \mathrm{L}$ \\
Antibiotic (Antimycotic 2\%) & $1 \mathrm{~mL}$ \\
FSH & $100 \mu \mathrm{L}$ \\
LH & $10 \mu \mathrm{L}$
\end{tabular}

PFF: porcine follicular fluid, FSH: follicular stimulating hormone, LH: luteinizing hormone

Table 3 Composition of North Carolina State University-37 maturation media $(50 \mathrm{~mL})$

\begin{tabular}{lc}
\hline Constituent & Concentration \\
\hline Stock solution A & $36 \mathrm{~mL}$ \\
Stock solution B & $9 \mathrm{~mL}$ \\
PFF & $5 \mathrm{~mL}$ \\
Glucose & $0.0045 \mathrm{~g}$ \\
Sorbitol & $0.0984 \mathrm{~g}$ \\
B-ME stock solution & $50 \mu \mathrm{L}$ \\
L-Cysteine stock solution & $500 \mu \mathrm{L}$ \\
Antibiotic (Antimycotic 2\%) & $1 \mathrm{~mL}$ \\
dbcAMP stock solution & $200 \mu \mathrm{L}$ \\
PMSG (pregnant mare serum gonadotropin) & $10 \mu \mathrm{L}$ \\
hCG (human chronic gonadotropin) & $10 \mu \mathrm{L}$ \\
\hline
\end{tabular}

PFF: porcine follicular fluid

Polar body formation occurs after the COCs expand from the zona pellucida and the matured oocyte reaches metaphase II stage (Jamnongjit \& Hammes, 2005). This indicates successful in vitro maturation of the oocyte and extrusion of the first polar body (cytoplasmic formation) within the perivitelline space. This was evaluated using an Olympus Oosight ${ }^{\mathrm{TM}}$ inverted imaging system (Shinjuku-ku, Tokyo, Japan), observing for the presence of fully expanded COC cells and polar body extrusion between the zona pellucida and the cytoplasmic space of the oocyte.

The data were analysed in a Microsoft Excel spreadsheet and descriptive statistics were performed followed by single factor ANOVA. The percentages were expressed as mean \pm SE and the differences between the groups were considered significant when the $P$ value was $<0.05$.

\section{Results and Discussion}

The maturity rates and polar body formations were significantly higher $(P<0.05)$ for NCSU 37 with $85.9 \%$ and $81.9 \%$, respectively, and there were no significant differences for EGF and NCSU-23 media ( $P$ $>0.05$ ) as $77.1 \% ; 72.5 \%$ and $77.1 \% ; 72.7 \%$ were obtained, respectively, as shown in Table 4. 
Table 4 Mean ( \pm SE) effects of different in vitro maturation media on maturation rate and polar body formations

\begin{tabular}{lccc}
\hline Treatments $^{*}$ & Oocytes (No.) & Maturation rate (\%) & Polar body status (\%) \\
\hline EGF (T1) & & & \\
NCSU-23 (T2) & 284 & $77.1( \pm 0.76)^{\mathrm{a}}$ & $72.5( \pm 0.64)^{\mathrm{a}}$ \\
NCSU-37 (T3) & 284 & $77.1( \pm 0.76)^{\mathrm{a}}$ & $72.7( \pm 1.61)^{\mathrm{a}}$ \\
& 284 & $85.9( \pm 1.13)^{\mathrm{b}}$ & $81.9( \pm 0.89)^{\mathrm{b}}$
\end{tabular}

*The treatment groups consisted of Treatment 1: epidermal growth factor (EGF), Treatment 2: NCSU-23; Treatment 3: NCSU-37

${ }_{a, b}$ Row means with different superscripts differ significantly at $P<0.05$

Maturation of immature and mature fully expanded cumulus-oocyte complex was evaluated under a light microscope as indicated by Figure 1, images A - D.

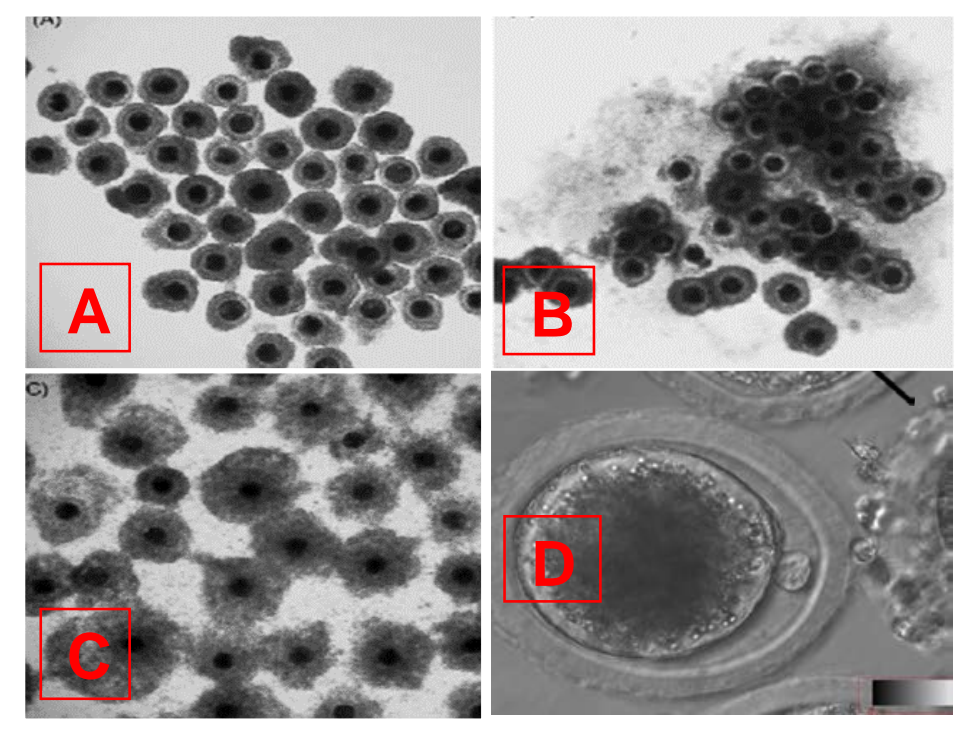

Figure 1 (A) Light microscope illustrating fully expanded cumulus-oocyte complex matured in Epidermal Growth Factor after 48 hrs; (B) Cumulus-oocyte complex matured in NCSU-23 after 48 hrs; (C) Cumulusoocyte complex matured in NCSU-37 after 48 hrs; (D) First polar body formation indicated by the arrow

The maturity rates and polar body formations in EGF and NCSU-23 media were not significantly different. However, the results for media NCSU-37 were highest and significant compared with the other maturation media.

Maturation media used in this study differ in terms of supplementation. EGF was supplemented with $10 \%$ BSA (bovine serum albumin), NCSU-23 was supplemented with hormones FSH (follicle stimulating hormone) and LH (luteinizing hormone), and NCSU-37 was supplemented with gonadotropins PMSG (pregnant mare serum gonadotropin) and hCG (human chronic gonadotropin). These gonadotropins have been reported to aid in the expansion of cumulus cells (Sutton et al., 2003), which allows for meiotic competency and increases the chances of further embryonic development. The addition of $\beta$-mercaptoethanol and L-Cysteine results in elevated oocyte glutathione regulating cysteine concentrations between granulosa cells, which assists in cumulus expansion, resulting in oocyte maturation. IVM media with beneficial factors such as EGF together with beta-mercaptoethanol and hypotaurine can enhance blastocyst rates (Mizushima \& Fukui, 2001), leading to successive embryo harvests.

Hormones FSH and LH play a role in inducing a transient increase in cyclic denosine monophosphate and stimulating EGF peptides, respectively. This results in an increase of the gap junction between oocytes and granulosa cells (Wang et al., 2013) facilitating COC expansion. Furthermore, this occurrence controls 
the activities of PKA (protein kinase A) and GPR3 (G protein-coupled receptor 3), resulting in an inhibitory phase before inducing meiotic maturation.

Luteinizing Hormone (LH) has been demonstrated to increase intracellular calcium oscillations levels, which will be more of a contribution towards induced meiosis leading to cleavage development (Wang et al., 2013), thus allowing subsequent embryo development, which is more beneficial to further embryonic competency.

Although the addition of FSH increases pentose phosphate pathway (PPP) activity, leading to increased COC expansions, LH reduces this pathway activity (Zuelke \& Brackett, 1992; Downs \& Utecht, 1999) concerning supplementations of hormones in the NCSU-23 media. The presence of glutamine in NCSU-23 could prove detrimental to maturation as it was reported to initiate germinal vesicle break down, but not meiotic completion (Downs \& Hudson, 2000). This block is also because of reduced activity of PPP, which utilizes intermediates for extracellular matrix expansion (Wang et al., 2013). The addition of BSA protein in IVM media has been shown to contain steroids such as estradiol at levels that are adequate for desirable cytoplasmic and nuclear maturation, though its use as a supplement is disconcerting as it may contain indeterminate contaminants (Mingoti et al., 2002).

Other components that are added to the NCSU-23 and NCSU-37, media such as follicular fluid, which is used widely as a replacement for serums, have been shown to influence oocyte maturation (Kim et al., 1996).

This study indicated that NCSU-37 had the highest rates of matured oocytes compared with EGF and NCSU-23 media.

The rate of maturation was higher than that of polar body formations owing to the removal of cumulus cells to identify the first polar body extrusion within the zona-pellucida of the oocyte. This was performed by vortex, which leads to the damage or loss of cells.

\section{Conclusion}

In conclusion, EGF and NCSU-23 had lower rates of maturation and meiotic development owing to their dissimilarities in composition. However, NSCU-37 rendered the highest results because the composition of the media contained beneficial constituents. Innovative assisted reproductive programs may be important for commercial farming systems, as this may facilitate production. In addition, it is important to conserve indigenous genetic resources.

\section{Acknowledgements}

Acknowledgements are extended to Germplasm Conservation and Reproductive Biotechnologies, ARC-API staff. Thanks are extended to Enterprise Tiger Brands Abattoir of Pretoria, Olifantsfontein, for providing the ovaries. This research was supported by Agricultural Research Council and the NRF.

\section{Authors' Contributions}

The study was conceived by GMP, based on the BTech study by GMP, which was produced under the supervision of TLM and AM. AM oversaw the experimental design for the preparation of the study and TLM suggested the method of analysis of the data. MMS contributed to editing the manuscript. All authors read and approved the final manuscript.

\section{Conflict of Interest Declaration}

The authors declare that they have no conflicts of interest with regard to this work.

\section{References}

Bailekae, M.M., 2012. Development of cryopreservation strategies for improved reproductive competence in South African pig genotypes. M.Tech. dissertation, Central University of Technology, Bloemfontein.

Bolling, L.C., 2001. The effect of growth hormone on pig embryo development in vitro and an evaluation of spermmediated gene transfer in the pig. MSc. VSc. Thesis. Graduate School of Virginia Polytechnic Institute, USA.

Brüssow, K.P., Tornera H., Kanitza, W. \& Rátkyb, J., 2000. In vitro technologies related to pig embryo transfer. Repr. Nutr. Dev. 40, 469-480.

Downs, S.M. \& Utecht, A.M., 1999. Metabolism of radiolabelled glucose by mouse oocytes and oocyte cumulus-cell complexes. Bio. Repr. 60, 1446-1452.

Downs, S.M. \& Hudson, E.D., 2000. Energy substrates and the completion of spontaneous meiotic maturation. Zygote 8 , 339-351.

Funahashi, H. \& Day, B.N., 1997. Advances in in vitro production of pig embryos. J. Repr. Fert. Supp. 52, $271-283$.

Grupen, C.G., 2014. Review: Current status of porcine embryo in vitro production. Camden, University of Sydney, Australia.

Halimani, T.E., Muchadeyi, F.C., Chimonyo, M. \& Dzama, K., 2010. Pig genetic resource conservation: The Southern African perspective. Ecological Economics 69, 944-951. 
Jamnongjit, M. \& Hammes, S.R., 2005. Oocyte maturation: The coming of age of a germ cell. Seminars in Reproductive Medicine 23, 234-241.

Kim, K.S., Mitsumizo, N., Fujita, K. \& Utsumi, K., 1996. The effects of follicular fluid on in vitro maturation, oocyte fertilization and the development of bovine embryos. Theriogenology 45, 787-799.

Mattioli, M., Galeati, G., Bacci, M.L. \& Seren, E., 1988. Follicular factors influence oocyte fertilizability by modulating the intercellular cooperation between cumulus cells and oocyte. Gam. Res. 21, 223-232.

Mingoti, G.Z., Garcia, J.M. \& Rosa-e-Silva, A.A., 2002. Steroidogenesis in cumulus cells of bovine cumulus-oocytecomplexes matured in vitro with BSA and different concentrations of steroids. J. Anim. Repr. Sci. 69, 175-186.

Mizushima, S. \& Fukui, Y., 2001. Fertilizability and developmental capacity of bovine oocytes cultured individually in a chemically defined maturation media. Theriogenology 55, 1431-1445.

Nagai, T., Funahashi, H., Yoshioka, K. \& Kikuchi, K., 2006. Update of in vitro production of porcine embryos. Front. Biosci. 11, 2565-2573.

Nedambale, T.L., Chokoe, T.C. \& Maiwashe, A., 2010. Status of Farm Animal Genetic Resources in South Africa. Animal Conservation Program in Emerging Economies. International Strategic Program for Conservation of Animal Genetic Resources for Food and Agriculture.

Petters, R.M. \& Wells, K.D. 1993. Culture of pig embryos. J. Repr. Fert. Supp. 48, 61-73.

Sutton, M.L., Gilchrist, R.B. \& Thompson, J.G., 2003. Effects of in-vivo and in-vitro environments on the metabolism of the cumulus oocyte complex and its influence on oocyte developmental capacity. Hum. Repr. Upd. 9, 35-48.

Vatzias, G. \& Hagen, D.R., 1999. Effects of porcine follicular fluid and oviduct conditioned media on maturation and fertilization of porcine oocytes in vitro. Biol. Reprod. 60, 42-48.

Wang, Q., Zhao, M., Jin, Y., Kim, N. \& Cui, X., 2013. Gonadotropins improve porcine oocyte maturation and embryo development through regulation of maternal gene expression. J. Embr. Trans. 28, 361-371.

Zuelke, K.A. \& Brackett, B.G., 1992. Effects of luteinizing hormone on glucose metabolism in cumulus-enclosed bovine oocytes matured in vitro. J. Endo. 131, 2690-2696. 